\title{
ORGANES DE DÉFENSE TÉGUMENTAIRES DES HYALINIA
}

\author{
PAR \\ Emile ANDRÉ \\ Premier assistant d'anatomie comparée. Université de Genève.
}

Avec la planche 32 .

Dans son beau travail sur les téguments des Pulmonés ${ }^{1}$, LEYDig consacre quelques lignes à une curieuse formation tégumentaire chez les Hyalinia. Le savant naturaliste allemand considérait ces formations comme des cellules mucipares dont le contenu peut s'étirer en un fil de byssus ou en un filament urticant. Voici du reste comme il s'exprime à ce sujet : « ...bei Hyalinia cellaria, allwo sich schon in der Haut des Rückens für die Luppe und selbst für's freie Auge aus dem dunkeln bläulich schimmernden Hautpigmente die Schleimdrüsen sehr scharf abheben, zeigen sie sich unter dem Mikroskop durch ihren Inhalt als höchst scharf gerundete, ovale, helle Körper. Und dieser Inhalt erscheint entweder als reine homogene Masse, oder er zerlegt sich in cylindrisch gekrümmte Züge und nach Einwirkung von Reagentien, etwa von chromsaurem Kali nimmt sich die Masse aus wie ein langer Nesselfaden, oder ein zu einem Knäuel zu-

${ }^{1}$ Leydig. Die Hautdecke und Schale der Gastropoden. Arch. für Naturgesch., 42 Jahrg. I, 1876, S. 225. 
sammen geschobener Byssusfaden. - Auch die vorhin erwähnten feinen spiraligen Linien an der hellen Inhaltsmasse der Drüsen mögen wohl auf eine fadige Sonderung hindeuten. »

Dans une petite note ${ }^{1}$ sur des cryptes épithéliales qu'on rencontre chez les Hyalinia, nous avons signalé en passant ces éléments que nous prenions également pour des cellules à mucus. Plus tard, nous en avons donné une description sommaire ${ }^{2}$ que nous venons maintenant compléter.

Pour l'étude de ces formations tégumentaires, nous nous sommes adressé à l'Hyalinia cellaria Gray. Grâce à l'amabilité de M. P. Pallary, professeur à Oran, auquel nous exprimons ici nos plus vifs remercîments, nous avons également pu faire porter nos recherches sur une espèce africaine, Hyalinia cheliella Pecchiol. Chez cette dernière, nous avons trouvé la structure histologique des téguments à peu de chose près identique à celle des $H$. cellaria.

Nous considérons ces formations tégumentaires comme des organes de défense et c'est pour cela que nous les appellerons

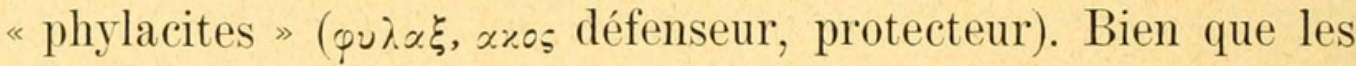
phylacites aient quelques analogies avec certains nématocystes, nous avons été obligé de créer un nouveau terme pour les désigner, parce que nous n'avons trouvé, dans la terminologie zoologique, aucun mot pouvant se rapporter à ces formations.

Avant de décrire en détail la structure et le fonctionnement des phylacites, nous rappellerons en quelques mots ce que nous en avons dit dans la note préliminaire que nous leur avons consacrée. Les phylacites sont des éléments défensifs localisés dans une région plus ou moins étendue des téguments du dos chez les Hyalinia. Ils se forment sous l'épithélium, dans des cellules ar-

${ }^{1}$ AndrÉ. Sur les téguments du Zonites cellarius. Zoologischer Anzeiger, No $411,1893$.

${ }^{2}$ André. Organes de défense tégumentaires chez le Zonites (Hyalinia) cellarius Gray. Ibid. No 564, 1898. 
rondies, analogues aux éléments du tissu conjonctif sphéroïdal. A l'état de repos, ils se composent d'un corps arrondi formé de couches concentriques. Lorsque l'Hyalinia les expulse pour sa défense, la partie centrale du phylacite fait saillie à l'extérieur, et le phylacite prend alors la forme d'un champignon.

Les phylacites, disons-nous, se trouvent dans la partie médiane de la paroi dorsale du corps des Hyalinia, dans sa région postérieure, partiellement recouverte par le manteau (même lorsque l'animal est en marche), en arrière de la portion des téguments qui possède des cryptes épithéliales. Le territoire occupé par les phylacites varie d'un individu à l'autre par la forme de ses contours et par ses dimensions. Nous avons représenté (fig. 1) la face dorsale d'une Hyaliniı cellaria, montrant, limitée par un trait rouge, la région contenant des phylacites; les points rouges indiquent les cryptes épithéliales. Examinée à un plus fort grossissement, la portion de la peau contenant des phylacites se montre sous l'aspect représenté par la fig. 4 .

En dehors de la région où les phylacites forment au-dessous de l'épithélium une couche assez compacte, on trouve souvent des cellules à phylacites (nous les appellerons dorénavant «phylacoblastes ») isolées, et cela jusque sur les côtés du pied. En examinant notre fig. 2 , on peut aussi se rendre compte de la distribution des phylacites dans les téguments des Hyalinia. Chez l'H. cheliella, le territoire occupé par les phylacites est généralement moins étendu que chez $H$. cellaria.

Examiné sur le frais, lorsqu'il n'a pas encore fonctionné et qu'il est encore contenu dans la cellule qui lui a donné naissance ( $d$, fig. 8), le phylacite se présente sous la forme d'un corps arrondi ou ovoïde, composé d'une substance transparente, incolore ou très légèrement jaunâtre, plus ou moins granuleuse. A l'intérieur, on aperçoit une vésicule (b) ronde, ovoïde ou quelquefois fusiforme, contenant elle-même des sphérules réfringentes $(c)$, en nombre variable (de trois à une vingtaine), dont le 
contenu est également granuleux. Lorsque le phylacite sera expulsé et prendra la forme d'un champignon (fig. 12, 14, 15 et 16) - nous verrons plus loin par quel procédé - sa partie externe ( $a$, fig. 8) deviendra ce que nous appellerons la tête du phylacite, sa partie interne (b) en donnera la tige et les sphérules réfringentes (c) deviendront ce que nous nommerons les vésicules piriformes. Les dimensions des phylacites sont éminemment variables; les plus gros que nous ayons observés mesuraient $0,11 \mathrm{~mm}$. dans leur plus grand diamètre.

Sur des coupes d'Hyalinia colorées par une teinture de carmin, les phylacites se détachent en rouge très foncé sur les tissus qui les environnent (fig. 2, 3, 9 et 13). Ils ont donc une grande affinité pour les teintures de carmin; mais cette affinité n'est pas également forte pour toutes les parties qui composent le phylacite : lorsqu'on décolore une coupe traitée par une teinture de carmin, on observe que ce sont les vésicules piriformes qui résistent le plus à l'action du réactif décolorant (c, fig. 10). Nous ajouterons que, même en employant la décoloration, il est assez difficile de distinguer, sur des coupes, la structure intime du phylacite.

Les phylacites sont formés et contenus dans des cellules rondes, analogues aux cellules conjonctives sphéroïdales (fig. 5, 9 et $13, d$ ) réparties au-dessous de l'épithélium externe. Chez les Hyalinia adultes, il n'y a qu'une seule couche de phylacoblastes (fig. 2), tandis que chez les jeunes il y a quelquefois deux ou trois couches de ces éléments (fig. 3), à différents stades de développement.

Dans le phylacoblaste qui lui a donné naissance, le phylacite est contenu dans une vacuole (fig. 8 e, fig. 9 c, fig. $10 d$ et fig. $13, b$ ). Cette dernière occupe une portion plus ou moins importante de la cellule et est remplie plus ou moins complètement par le phylacite. Lorsque le phylacite est encore peu développé (a, fig. 9), la vacuole (c) est petite relativement à la cellule 
entière, tandis que, pour un phylacite ayant atteint tout son développement, la vacuole peut occuper la totalité de la cellule (e, fig. 8). Cette dernière, qui a perdu graduellement son noyau par résorption, est alors réduite à sa simple membrane. Le phylacoblaste ne forme plus alors qu'un sac à paroi mince (fig. 8 et 11) dans lequel on ne retrouve rien de ce qui constituait la cellule primitive, si ce n'est la membrane.

La constitution du phylacite au repos étant connue, examinons maintenant ce qu'il devient lorsqu'il est expulsé et comment s'opère cette expulsion. Lorsqu'on racle avec un scapel les téguments du dos d'une Hyalinia tuée par asphyxie sous l'eau ou par immersion dans l'eau bouillante, et qu'on examine au microscope le produit de ce raclage, on y remarque un grand nombre de corps ayant la forme de champignons (fig. 12, 14 et 15). Ce sont les phylacites dont la vésicule interne ( $b$, fig. 8) a déchiré la zone externe (a) et a fait saillie à travers cette déchirure en s'allongeant en forme de tige. Le chapeau du champignon (a, fig. 15) correspond donc à la zone externe du phylacite ( $a$, fig. 8 ) et sa tige ( $b$, fig. 15 ) à la vésicule interne ( $b$, fig. 8 ).

L'expulsion de la vésicule interne du phylacite pour former la tige s'opère certainement sous l'action des fibres musculaires qui se trouvent dans la couche musculo-conjonctive sous-épithéliale des téguments du dos. Lorsque le phylacite est comprimé par ces faisceaux musculaires, surtout dans sa partie profonde, sa vésicule interne se porte vers la partie la moins comprimée du phylacite, c'est-à-dire celle qui est la plus voisine de la surface des téguments, et, la compression augmentant, elle déchire la zone externe et sort par cette déchirure en s'allongeant. Le phylacite prend alors la forme représentée fig. 15. Une comparaison un peu triviale nous aidera à nous faire comprendre: lorsqu'on serre une cerise entre les doigts, le noyau de celle-ci ne tarde pas à déchirer la pulpe du fruit et à sortir par cette ouverture; si le noyau était un corps plastique, pouvant s'allon- 
ger en sortant par cet orifice, et s'il était retenu d'une façon quelconque à la partie charnue de la cerise, on aurait alors un corps qui présenterait une grande analogie de forme avec un phylacite expulsé.

Pour la même raison, les sphérules ( $c$, fig. 8), que nous appellerons les «vésicules piriformes, » se rapprocheront de l'extrémité libre de la tige où elles finiront par déboucher et se videront de leur contenu ( $c$, fig. 10, 12, 14 et 16). Dans la fig. 10, on voit les vésicules piriformes s'étirer et diriger leur col vers l'extrémité de ce qui deviendra la tige du phylacite; dans cette préparation, la décoloration a été poussée jusqu'à ce que les vésicules piriformes seules restent colorées. Il est à supposer que les vésicules piriformes constituent la partie active, vraiment défensive du phylacite et que leur contenu a des propriétés toxiques ${ }^{1}$.

L'expulsion de la vésicule interne du phylacite pour former la tige est très rapide; en tout cas, cette opération ne s'arrête presque jamais à mi-chemin, et dans le produit de la dilacération des téguments du dos des Hyalinia, on ne trouve que des phylacoblastes clos (fig. 8) et contenant encore leur phylacite et des phylacites ayant expullsé leur tige et leurs vésicules piriformes. Quelquefois cependant, sur des coupes, on rencontre des phylacites dont la tige n'est pas complètement extériorée (fig. 10).

Lorsque le phylacite a émis sa tige, on observe alors facilement la structure en couches concentriques de sa tête ( $a$, fig. 12 et 16), structure qui n'était que peu ou pas perceptible sur le phylacite au repos, lorsqu'il était encore contenu dans la vacuole

1 Les Hyalinia cheliella répandent, lorsqu'on les moleste, une odeur très forte, identique à celle que répand la chenille du Cossus ligniperda et, lorsqu'on les met dans l'eau, on voit se rassembler à la surface du liquide de très fines gouttelettes de l'huile essentielle qui produit cette odeur. Nous n'avons pas pu déterminer d'une façon certaine si cette essence est sécrétée par les phylacoblastes, par les cryptes épithéliales ou par les glandes du manteau.. 
du phylacoblaste. Dans quelques cas (fig. 14), la structure granuleuse de la tête cache la disposition en couches concentriques. La fig. 6 qui représente une coupe d'une tige qui s'est séparée de la tête en emportant des lambeaux de cette dernière, montre également bien ces strates concentriques. Ce fait que la tige entraîne avec elle des portions de la tête, se présente assez fréquemment; on en a un exemple dans la fig. 12. Le même cas s'est produit pour le phylacite représenté par la fig. 7 ; la zone externe, la tête, s'est étirée lors de la formation de la tige et des lambeaux en ont été arrachés par cette dernière; la tige s'est même séparée complètement de la tête.

Comme on le voit d'après nos fig. $12,14,15$ et 16 , les dimensions relatives de la tête et de la tige sont variables. Le plus souvent la tête est plus volumineuse que la tige, mais quelquefois cependant c'est le contraire qui s'observe et la tige peut sortir d'une tête très réduite (fig. 16). On voit aussi que les dimensions et le nombre des vésicules piriformes sont variables.

Lors de l'expulsion du phylacite hors de la cellule qui lui a donné naissance, les parois de celle-ci se rompent pour lui livrer passage (fig. 10). Les cellules de l'épithélium s'écartent ensuite et laissent émerger entre elles le phylacite. La déchirure de la paroi du phylacoblaste est de forme variable (fig. 5), mais elle s'effectue toujours, comme c'était à supposer, dans la portion qui est en contact avec l'épithélium externe. La fig. 5 représente cinq phylacoblastes ayant expulsé leur phylacite; comme on le voit, la forme et les dimensions de la déchirure sont très variables.

Ainsi que nous le disions plus haut, les phylacites prennent naissance dans des éléments (fig. 9) analogues aux cellules conjonctives sphéroïdales. Il se creuse une petite vacuole au sein du corps cellulaire et c'est dans cette vacuole que nait le phylacite. Ensuite, vacuole et phylacite augmentent petit à petit de volume jusqu'à complet développement. 
Dans les jeunes phylacites, on ne peut rien distinguer de leur structure intime; aussi n'est-il pas possible de dire si le phylacite s'accroît par intussusception, si la vésicule centrale qui donnera la tige se forme la première, et si la couche externe qui deviendra la tête vient se déposer sur elle, ou bien si la formation de ces deux parties se fait par différenciation graduelle.

La fig. 9 montre que le même phylacoblaste peut fonctionner deux fois et qu'il peut produire successivement deux phylacites; mais ce cas est plutôt l'exception. On ne rencontre pas fréquemment non plus, même chez de jeunes Hyalinia, des phylacoblastes en voie d'élaborer leur phylacite. Cela nous laisse supposer que les Hyalinia n'usent des phylacites pour leur défense que dans des cas exceptionnels. Les phylacoblastes que l'on rencontre le plus souvent, sur des coupes et dans le produit de la dilacération des téguments du dos, sont ceux des types représentés par les fig. 8 et 13 : le corps cellulaire du phylacoblaste est nul ou à peu près, le noyau est atrophié ou même a totalement disparu, la cellule est réduite à sa seule membrane.

On trouve quelquefois chez les Hyalinia adultes des phylacoblastes (fig. 11) réduits à l'état de simple sac, dont l'intérieur contient un grand nombre de corpuscules arrondis présentant la même affinité pour le carmin que les phylacites normaux. Nous supposons qu'on se trouve alors en présence de phylacites en dégénérescence qui, n’ayant pas été utilisés, se résorbent petit à petit en se fragmentant.

Quant à la nature chimique des phylacites, elle nous est inconnue. En tout cas, nous pouvons dire qu'ils ne sont pas composés de mucus; en effet, traités par les teintures d'hématoxyline, ils ne se colorent pas, tandis que l'on sait que tous les éléments contenant de la mucine ont une grande affinité pour la matière colorante du bois de Campêche. Le carmin, par contre, colore les phylacites en rouge foncé. Traités par la solution d'iode dans l'iodure de potassium, ils se colorent très fortement. 
Sous l'action de l'acide azotique, ils deviennent jaune verdâtre clair.

La description que nous venons de donner des phylacites nous montre qu'ils ont certaines analogies avec les nématocystes; non pas précisément par leur structure, mais par leur mode de développement et par la façon dont ils fonctionnent. Cela nous a engagé à considérer ces curieux éléments, jusqu'à plus ample information, comme des organes de défense. 
Fig. 1. Hyalinia cellaria. Peau du dos, vue de champ, sur le frais. Le trait rouge circonscrit la région où se trouvent les phylacites; les points rouges indiquent des cryptes épithéliales. Gross. כ̆ fois.

2. $)$

$3 . \quad 》$

4. )

วั. 》

" $6 . \quad)$

1) $7 . \quad$

8. )

Coupe transversale de l'animal entier ; $a$, couche des phylacites (en rouge); $b$, cryptes épithéliales. Gross. 18 fois.

Coupe transversale de la peau du dos; $a$, phylacite (en rouge); b, épithélium externe; $c$, pigment; $d$, masse musculo-conjonctive. Gross. 70 fois.

Peau du dos, vue de champ ; l'épithélium externe est enlevé ; $a$, phylacite ; $b$, pigment. Gross. 70 fois.

Peau du dos, vue de champ; l'épithélium externe est enlevé. On voit כ̆ phylacoblastes ayant expulsé leur phylacite; $a$, noyau du phylacoblaste; $b$, déchirure par laquelle est sortie le phylacite. Gross. 170 fois.

Coupe optique longitudinale (schématisée) d'une tige de phylacite; $a$, tige ; $b$ et $c$, lambeaux des couches concentriques formant la tète du phylacite. Gross. 320 fois.

cheliella. Tète d'un phylacite complètement séparé de sa tige. Gross. 235 fois.

Phylacite encore contenu dans le phylacoblaste; $a$, partie qui deviendra la tète du phylacite; $b$, partie qui formera la tète ; $c$, vésicules piriformes dont les cols ne sont pas encore formés; $d$, membrane cellulaire; $e$, vacuole. Gross. 300 fois.

) $9 . \quad$ )

cellaria. Phylacoblaste contenant un phylacite déjà formé ( $a$ ) et un autre (b) en voie de formation; $c$, vacuole; $d$, noyau. Gross. 390 fois.

10. )

cheliella. Portion d'une coupe transversale de la peau du dos montrant un phylacite émergeant de l'épithélium. La paroi de la cellule (e) est déchirée; $a$, tète; $b$, tige ; $c$, vésicule piriforme ; $d$, vacuole ; $e$, membrane cellulaire; $f$, épithélium 
externe. La décoloration a été poussée jusqu’à ce que les vésicules piriformes seules restent colorées. Gross. 300 fois.

Fig.11. Hyalinia cellaria. Phylacoblaste en dégénérescence. Gross.400 fois. ) 12. " " Phylacite expulsé; $a$, tête; $b$, tige ; $c$, vésicules piriformes. Gross. 280 fois.

"13. " " Phylacoblaste, sur une coupe ; $a$, phylacite; $b$, vacuole; $c$, noyau; $d$, cellule conjonctive; $e$, épithélium externe; $f$, pigment. Gross. 390 fois.

") 14. " cheliella. Phylacite expulsé ; $a$, tête; $b$, tige ; $c$, cols des vésicules piriformes. Gross. 300 fois.

) 1รั. " cellaria. Phylacite expulsé; $a$, tête; $b$, tige. Gross. 170 fois.

) 16. " " Phylacite expulsé ; $a$, tète ; $b$, tige ; $c$, vésicules piriformes. Gross. 280. 


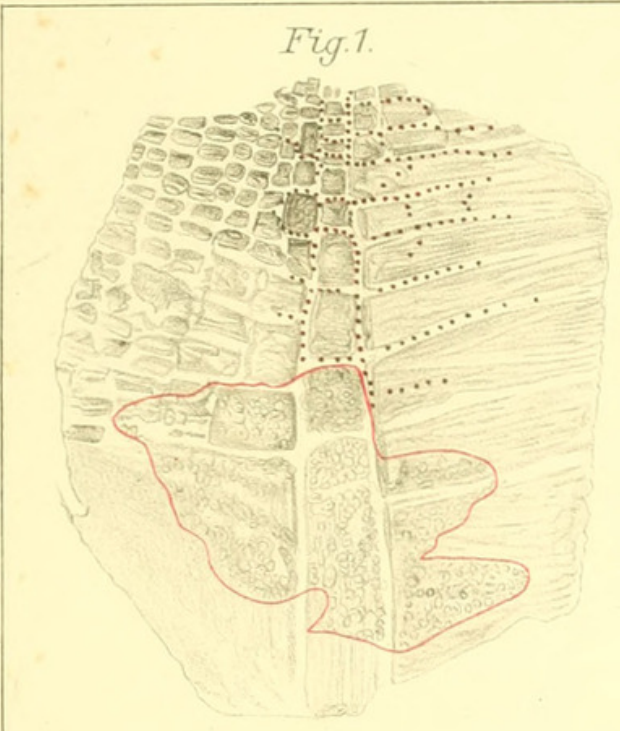

Fig.5.
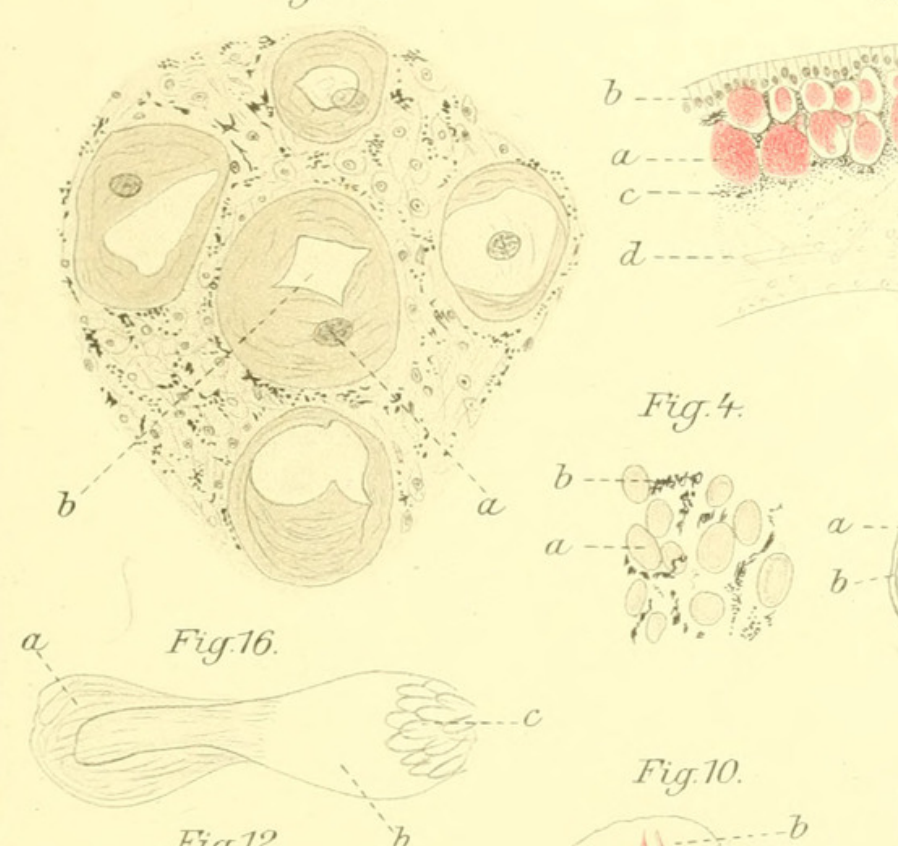

Fig.3
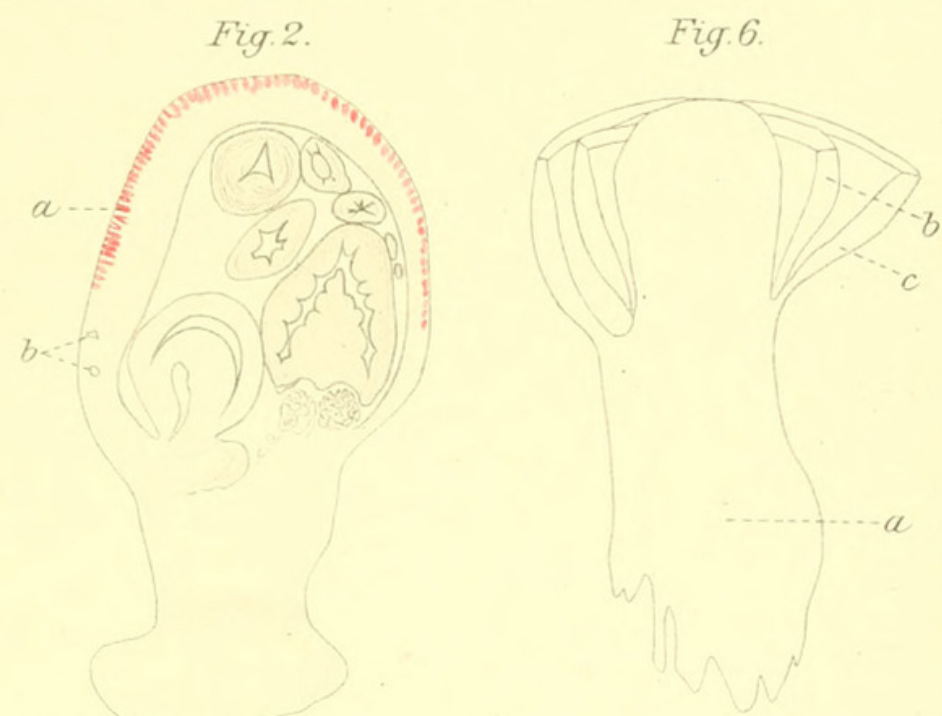

Fig.7.

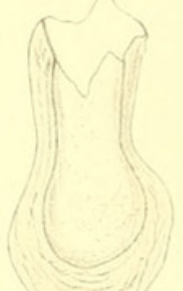

Fig.8.

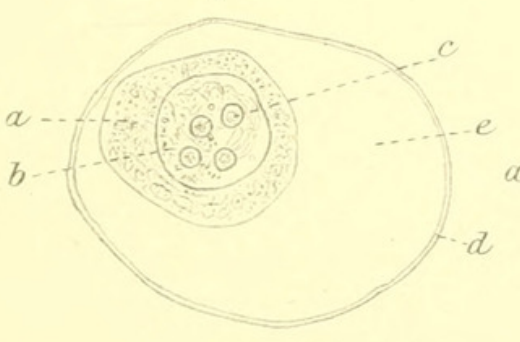

Fig. 9.

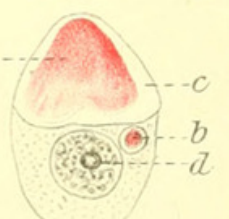

Fig. 10 .

Fig.11.
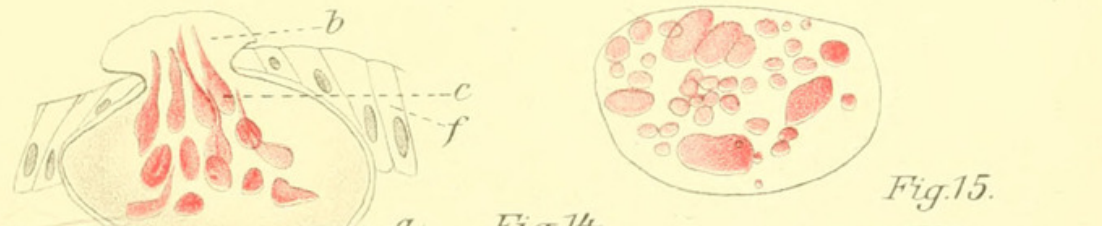

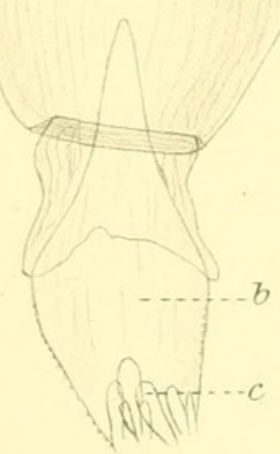

Fig.74.

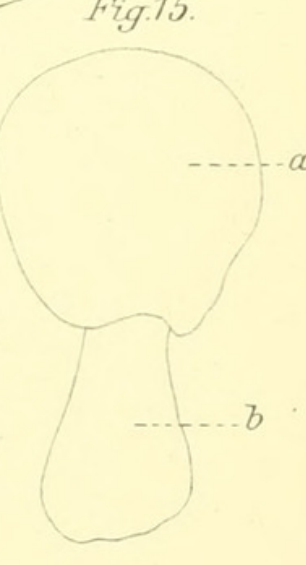

E. André del.

Iith.Anstr EA Funke, Leipzig.

E. André. Hyalinia. 


\section{$2 \mathrm{BHL}$ Biodiversity Heritage Library}

André,

$\mathrm{E}$

mile. 1900. "Organes de défense tégumentaires des Hyalinia." Revue suisse de zoologie 8(3), 425-433. https://doi.org/10.5962/p.318256.

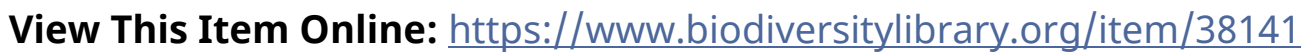

DOI: https://doi.org/10.5962/p.318256

Permalink: https://www.biodiversitylibrary.org/partpdf/318256

\section{Holding Institution}

MBLWHOI Library

\section{Sponsored by}

MBLWHOI Library

\section{Copyright \& Reuse}

Copyright Status: NOT_IN_COPYRIGHT

This document was created from content at the Biodiversity Heritage Library, the world's largest open access digital library for biodiversity literature and archives. Visit BHL at https://www.biodiversitylibrary.org. 